$\underline{\text { Preprint typeset in JHEP style - HYPER VERSION }}$

TIFR/TH/11-45

\title{
Unravelling the novel Higgs mechanism in $(2+1) d$ Chern-Simons theories
}

\author{
Sunil Mukhi* \\ Tata Institute of Fundamental Research, \\ Homi Bhabha Rd, Mumbai 400 005, India
}

\begin{abstract}
Chern-Simons gauge theories in $2+1$ dimensions with multiple gauge fields exhibit novel properties that are analysed here in some detail. A striking feature is the possibility of a non-propagating Chern-Simons field acquiring a massless propagating mode via a Higgs mechanism. This novel Higgs mechanism, originally discovered in the context of M-theory, is studied here without reference to M-theory or supersymmetry. It is revealed as a variant of topological mass generation and shown to arise only when Chern-Simons and mass matrices are not simultaneously diagonalisable. Sufficient conditions are found for it to occur. It is speculated that some analogue of the NHM could occur in theories of condensed-matter systems similar to those exhibiting the fractional quantum Hall effect, as well as in $2+1$ dimensional gravity.
\end{abstract}

Keywords: Gauge theory, Chern-Simons theory, Higgs mechanism.

\footnotetext{
${ }^{*}$ E-mail: mukhi@tifr.res.in
} 


\section{Contents}

1. Introduction

2. Topological mass and novel Higgs mechanism 3

2.1 Topologically massive theories 3

2.2 Multiple fields 5

2.3 Necessary and sufficient conditions: two-field case 8

3. General number of fields 12

3.1 Non-diagonalisability: a necessary condition 12

3.2 Sufficient conditions: three field case 14

3.3 General case 15

3.4 Higgs fields 16

4. Non-abelian case 17

4.1 Difference Chern-Simons 17

$4.2 B \wedge F$ theory 21

5. Potential applications 21

5.1 Condensed-matter systems 22

$5.2(2+1)$ d gravity 25

\section{Introduction}

Three-dimensional gauge theories have some special features, notably the possibility of writing a Chern-Simons kinetic term that is first-order in the derivatives [1, 2, 3]. In addition to this term, a $(2+1)$ d gauge theory can have a conventional Maxwell kinetic term as well as an explicit mass term that typically arises via a Higgs mechanism. The possibility of three distinct types of terms in the Lagrangian for free gauge fields makes for a more subtle spectrum than is familiar in four-dimensional field theory, where a free gauge field can only have a Maxwell or mass term. In particular, parity violation is a generic feature of theories with a Chern-Simons term. 
If one now extends this structure to multiple species of gauge fields, new subtleties arise. One of the most striking of these is a new type of Higgs mechanism wherein a non-propagating gauge field absorbs the degree of freedom of a Higgs field and turns into a massless propagating gauge field [4]. This phenomenon, the "novel Higgs mechanism" (NHM), was discovered and usefully applied to study the moduli space of extended superconformal field theories describing multiple membranes in M-theory, namely the BLG and ABJM field theories. Despite its origin, the mechanism itself does not rely on supersymmetry or string/M-theory. It is a subtle but elementary feature of ordinary quantum field theory in $(2+1) \mathrm{d}$, and as such could be of interest in a much wider context. In particular, it might well have applications in condensed-matter systems, where Chern-Simons field theories are already known to play an interesting role (see for example [5, 6, 7, 8]). Notably the NHM occurs in systems that conserve parity [9, 10].

One purpose of this note is to analyse the spectrum of $(2+1) d$ gauge theories with multiple gauge fields. We will see that the novel Higgs mechanism arises for a very general class of such theories. Our analysis reveals that the NHM arises out of a conflict between simultaneous diagonalisability of the kinetic (Chern-Simons) terms and the mass terms. This conflict in turn arises when one has Chern-Simons terms of both signs. There is no analogue of this phenomenon in "normal" field theories with second-order kinetic terms, whose sign is determined by requiring the absence of negative-norm states. One consequence of non-diagonalisability in $(2+1) \mathrm{d}$ is that it is not straightforward to read off the spectrum. As a result the spectrum can have various unusual features and the NHM turns out to be just one of these.

A well-known characteristic of $(2+1)$ d gauge theories with a single gauge field, observed nearly three decades ago[3], is that Maxwell gauge fields in $2+1$ dimensions acquire a "topological mass" when a Chern-Simons interaction is added to the action. The propagating degrees of freedom have a single degree of freedom with spin +1 but no corresponding spin -1 state (the reverse holds if we change the sign of the mass parameter). Subsequently [11], it was noticed that the topologically massive action, containing Maxwell and Chern-Simons terms, is equivalent to a different action with a Chern-Simons term and an explicit mass term 12] but no Maxwell term. Both sides of this equivalence correspond to massive theories. We will see that the analogue of this duality in systems with multiple gauge fields provides a natural setting to understand the NHM. Namely, in appropriately chosen systems the analogous duality maps a set of Chern-Simons and mass terms to a Maxwell term without a mass. In turn, the original theory with Chern-Simons and mass terms in turn can descend from a conformal theory in which the mass terms are induced by a Higgs mechanism. This means a massless propagating Yang-Mills field can arise on the Higgs 
branch of a conformal field theory.

In the context of M-theory membranes the NHM was originally presented [4] in a nonAbelian form and the emphasis was on those features that are relevant to multiple membrane dynamics. However in the present work we first spell it out in an Abelian setting with Higgs fields carrying U(1) charges. Subsequently we introduce non-Abelian gauge fields with corresponding Yang-Mills and non-Abelian Chern-Simons terms, and discuss the role of interactions. Towards the end some possible applications of the results are briefly explored.

\section{Topological mass and novel Higgs mechanism}

\subsection{Topologically massive theories}

We start by reviewing the concept of topologically massive gauge theories in $2+1$ dimensions [3] and their duality 11] to a class of Chern-Simons theories with an explicit mass term, proposed in Ref. [12] where they were dubbed "self-dual actions". It will be seen that the novel Higgs mechanism is an extension of these theories and dualities to the case of multiple gauge fields, providing a natural setting for the phenomenon. Since the immediate goal is to understand the spectrum of excitations, we initially work at the linearised level, or equivalently in the Abelian theory.

The basic topologically massive theory is given by a Lagrangian for a 1-form Abelian gauge field $A$ with the Maxwell + Chern-Simons Lagrangian:

$$
\mathcal{L}_{1}=\frac{1}{2} d A \wedge^{*} d A-\frac{1}{2} m A \wedge d A
$$

The equations of motion of this theory are:

$$
d^{*} d A=m d A
$$

As explained in Ref.[3], this theory has a single on-shell degree of freedom that is massive and has spin +1 . Because parity is violated, it is possible to have no spin -1 mode.

Next consider a different Lagrangian consisting of a Chern-Simons term plus an explicit mass term:

$$
\mathcal{L}_{2}=\frac{1}{2} A \wedge d A+\frac{1}{2} m A \wedge^{*} A
$$

This is the self-dual Lagrangian of Ref.[12]. This time the equations of motion are:

$$
{ }^{*} d A=m A
$$

It is easy to verify that the theory of Eq. (2.3) is equivalent to that of Eq. (2.1). Classically, the equivalence is shown as follows. First,

$$
{ }^{*} d A=m A \quad \Longrightarrow \quad d^{*} d A=m d A
$$


so $\mathcal{L}_{2} \Longrightarrow \mathcal{L}_{1}$. For the converse,

$$
d^{*} d A=m d A \Longrightarrow d\left({ }^{*} d A-m A\right)=0 \Longrightarrow{ }^{*} d A-m A=d \lambda
$$

and a field re-definition $A \rightarrow A-\frac{1}{m} d \lambda$ gives Eq. (2.4). Thus $\mathcal{L}_{1} \Longrightarrow \mathcal{L}_{2}$. A detailed discussion of the quantum-mechanical equivalence of the two theories can be found in Ref. 11].

Let us make some comments on this equivalence.

1. Comparing the two Lagrangians Eq. 2.1) and Eq. (2.3) we see that the former is gauge-invariant while the latter does not seem to have a gauge symmetry. In fact, $A$ in the second Lagrangian can be thought of as the dual field strength ${ }^{*} d A$ of the first one, so the second Lagrangian actually expresses the field content of the first one in gauge-invariant variables. This is seen more clearly by introducing 11] a "master Lagrangian" involving two independent 1 -form fields $A$ and $f$ :

$$
\mathcal{L}_{\text {master }}=\frac{1}{2} f \wedge^{*} f+f \wedge d A-\frac{1}{2} m A \wedge d A
$$

The equation of motion of $f$ gives:

$$
f={ }^{*} d A
$$

while the equation of motion of $A$ is:

$$
d f=m d A
$$

Eliminating $f$ we recover Eq. (2.2), while eliminating $A$ (it is not algebraic, but only appears as $d A$ so it can still be eliminated between the two equations of motion) we find Eq. (2.4) for the field $f$.

2. While Eq. (2.1) has a smooth massless limit, Eq. (2.3) becomes purely topological and thereby loses a degree of freedom as $m \rightarrow 0$. Indeed the proof of equivalence between the two theories involves a gauge transformation that becomes singular as $m \rightarrow 0$ (see below Eq. (2.6)).

3. In $(2+1) \mathrm{d}$, the sign of the mass $m$ of a state of a spin- 1 field is meaningful and can be interpreted as follows: $m>1$ means the state has spin +1 and $m<1$ means it has spin -1 . For $m=0$ the little group is trivial and there is no concept of spin.

4. As observed in Ref.[13], in addition to gauge fields one can imagine having a scalar Higgs field in the system such that its vacuum expectation value generates the mass term in Eq. (2.3). Therefore the conversion of a Chern-Simons action into a topologically massive Yang-Mills action can be viewed as a kind of Higgsing.

5. The coefficient of an interacting Chern-Simons term corresponding to a compact gauge group is required to be quantised. We temporarily ignore this requirement as it 
makes no difference to the present discussion. It will be more important when non-Abelian gauge fields and interactions are introduced.

To complete this discussion it is useful to write down the spectrum of the most general free Lagrangian in $(2+1) \mathrm{d}$ involving a single gauge field, consisting of an arbitrary combination of all three terms: a Maxwell term, a Chern-Simons term and an explicit mass term. Thus, consider:

$$
\mathcal{L}=\frac{1}{2} w d A \wedge^{*} d A-\frac{1}{2} x A \wedge d A+\frac{1}{2} y A \wedge^{*} A
$$

Here $w, x, y$ are constants. While $w$ must be positive, $x, y$ can have either sign.

From the preceding discussion we know that for $y=0$ the theory has a single state of mass $x / w$, and for $w=0$ there is a single state of mass $-y / x$. For $x=0$ there is a

pair of parity-conjugate states of mass $\pm \sqrt{y / w}$ (which are tachyonic if $y<0$ ). Finally, a parity transformation $x \rightarrow-x$ should interchange the masses and spins of the two states, i.e. $m_{1} \leftrightarrow-m_{2}$. These considerations suffice to determine the spectrum of the generic theory (which can more directly be obtained by examining the propagator). We have two states of masses $\left(m_{1}, m_{2}\right)$ and $\operatorname{spins}\left(\operatorname{sign}\left(m_{1}\right), \operatorname{sign}\left(m_{2}\right)\right)$ given by:

$$
m_{1}=\frac{x+\sqrt{x^{2}+4 w y}}{2 w}, \quad m_{2}=\frac{x-\sqrt{x^{2}+4 w y}}{2 w}
$$

As $x \rightarrow 0$ one gets the expected parity-symmetric answer. For $w \rightarrow 0$ one of the masses goes to infinity and the other to the desired finite value. Finally for $y \rightarrow 0$ we find a massive state of the correct mass, and apparently an extra massless state. However in this limit gauge invariance is recovered and has the effect of decoupling the would-be extra massless state. As a result there is no massless state in the spectrum of the theory unless both the Chern-Simons and the mass term are absent, i.e. $x=y=0$.

\subsection{Multiple fields}

We now consider multiple 1-form fields in $(2+1) d$ with Chern-Simons and mass terms. We do not include Maxwell terms to start with. The reason, as indicated in the introduction, is that we would like to consider theories with an underlying conformal invariance broken only by a Higgs expectation value. Such a theory can have Chern-Simons terms and minimal couplings for the gauge fields, but no Maxwell terms.

Naively one would not expect to find any qualitatively new phenomena compared to what we have discussed in the previous subsection merely by introducing additional fields. After all, given a free field theory we usually diagonalise both the kinetic and mass terms before introducing interactions, and if this were possible in the presence of Chern-Simons 
terms then we would obtain a set of decoupled theories, each one of the form of Eq. 2.10) with $w=0$. There can be no massless propagating degrees of freedom in such a theory.

However, for $(2+1) \mathrm{d}$ theories with Chern-Simons and mass terms for multiple spin-1 fields, there is a potential conflict between diagonalisability of these two terms. As we will see shortly, this can lead to a qualitatively new feature: the possibility of a massless propagating state. This is what we call the novel Higgs mechanism (NHM). There are other interesting features that also arise for the first time when there are multiple fields.

To understand this issue consider a collection of fields $A^{I}, I=1,2, \cdots n$ described by the most general abelian Chern-Simons-mass Lagrangian (this can be generalised to include explicit Maxwell terms):

$$
\mathcal{L}=\frac{1}{2} k_{I J} A^{(I)} \wedge d A^{(J)}+\frac{1}{2} m_{I J} A^{(I)} \wedge^{*} A^{(J)}
$$

Both $k_{I J}$ and $m_{I J}$ are constant real symmetric matrices. $k_{I J}$ is taken to be non-degenerate, while $m_{I J}$ is allowed to have zero eigenvalues. The transformation:

$$
\delta A^{(I)}=d \Lambda^{(I)}
$$

is a gauge invariance for every set $\left\{\Lambda^{(I)}\right\}$ satisfying $m_{I J} \Lambda^{(J)}=0$, i.e. for every null eigenvector of $m$. Let us now try to bring this action into standard form as a sum of free actions for $n$ decoupled fields.

For comparison, we first recall how this is done for a generic free scalar field theory (in any dimension) with Lagrangian:

$$
-\frac{1}{2} g_{I J} \partial_{\mu} \phi^{I} \partial^{\mu} \phi^{J}-\frac{1}{2}\left(m^{2}\right)_{I J} \phi^{I} \phi^{J}
$$

where $\phi^{i}, I=1,2, \cdots, n$ are real scalar fields. Here $g_{I J}$ and $\left(m^{2}\right)_{I J}$ are constant real symmetric matrices and $g_{I J}$ is positive-definite (otherwise the theory has ghosts). To bring the Lagrangian into its standard form, one first performs an orthogonal transformation on $\phi^{I}$ to diagonalise $g_{I J}$, which then takes the form $\operatorname{diag}\left(g_{1}, g_{2}, \cdots, g_{n}\right)$ with $g_{I}>0$ for all $I$. Next one re-scales the fields:

$$
\phi^{I} \rightarrow \frac{\phi^{I}}{\sqrt{g_{I}}}
$$

so that the kinetic form has the identity metric $\delta_{I J}$. Finally one performs another orthogonal transformation on $\phi^{I}$ that diagonalises $m^{2}$ while preserving the kinetic term, ending up with:

$$
-\frac{1}{2} \partial_{\mu} \phi^{I} \partial^{\mu} \phi^{I}-\frac{1}{2} m_{I}^{2} \phi^{I} \phi^{I}
$$

Some of the $m_{I}$ can be equal to zero and we can also allow some to be imaginary (i.e. $\left.m_{I}^{2}<0\right)$ to allow for tachyons that are eventually stabilised by a potential. Thus the theory has been reduced to a collection of decoupled fields, some massive and others massless. 
When we try to apply the analogous procedure to Eq. 2.12), we find a rather different result. Upon diagonalising $k_{I J}$, it turns into $\operatorname{diag}\left(k_{1}, k_{2}, \cdots, k_{n}\right)$ but the eigenvalues $k_{i}$ are not required to be positive. The theory with negative eigenvalues, or both signs of eigenvalues, is perfectly consistent and - as is now well-known - field theories relevant to Mbranes [14, 15] have levels of both signs, which even permits parity to be conserved [10]. To be completely general, we therefore assume there are $p$ negative and $q$ positive eigenvalues with $p+q=n$. Since the $A^{(I)}$ are real, the best we can do after diagonalising $k_{I J}$ is to re-scale:

$$
A^{(I)} \rightarrow \frac{A^{(I)}}{\sqrt{\left|k_{I}\right|}}
$$

upon which the action Eq. (2.12) reduces to:

$$
\mathcal{L}=\frac{1}{2} \eta_{I J} A^{(I)} \wedge d A^{(J)}+\frac{1}{2} m_{I J} A^{(I)} \wedge^{*} A^{(J)}
$$

where $\eta_{I J}$ is a diagonal matrix with $p$ elements equal to -1 and the remaining $q=n-$ $p$ elements equal to +1 : the Lorentzian metric preserved by $O(p, q)$. Hence the linear transformations $A^{I} \rightarrow \Lambda_{I J} A^{J}$ which preserve the kinetic term are given by matrices $\Lambda_{I J}$ satisfying:

$$
\Lambda^{T} \eta \Lambda=\eta
$$

namely the $O(p, q)$ Lorentz transformations. The mass matrix can therefore be transformed only as:

$$
m \rightarrow \Lambda^{T} m \Lambda, \Lambda \in O(p, q)
$$

In general, a Lorentz transformation is not sufficient to diagonalise $m$.

We would now like to know under what conditions there exists a Lorentz transformation that diagonalises $m_{I J}$ in the basis where $k_{I J}$ is diagonal. Whenever this is possible, the theory will reduce to a collection of decoupled free fields with definite masses, and there will be no new phenomena such as the novel Higgs mechanism. The transformation law of the matrix $m_{I J}$ in Eq. (2.20) is that of a second-rank symmetric tensor under $O(p, q)$ Lorentz transformations. Therefore this is analogous to the question of whether the stressenergy tensor $T_{\mu \nu}$ of a field theory can be diagonalised by Lorentz transformations in a $p+q$-dimensional space of signature $(p, q)^{1}$.

We start by considering a general $O(p, q)$ matrix $\Lambda^{I}{ }_{J}$ which by definition satisfies:

$$
\Lambda_{K}^{I} \Lambda_{L}^{J} \eta_{I J}=\eta_{K L}, \quad \eta_{I J}=\operatorname{diag}\{\underbrace{-1,-1, \cdots,-1}_{p}, \underbrace{1,1, \cdots, 1}_{q}\}
$$

\footnotetext{
${ }^{1}$ I thank Nemani Suryanarayana for this observation and for initial collaboration on the analysis below.
} 
where the indices $I, J$ take the $p+q$ values $-p+1,-p+2, \cdots,-1,0,1, \cdots, q$, so that non-positive values label timelike directions. Let us now use $i, j, \cdots$ to label the spacelike directions $(1,2, \cdots q)$, and $m, n, \cdots$ to label the time-like directions $(-p+1,-p+$ $2, \cdots,-1,0)$. Define a set of $(p+q)$-component vectors $V_{(m)}^{I}=\Lambda_{m}^{I}$ and another set $W_{(i)}^{I}=\Lambda_{i}^{I}$. Clearly these vectors have the following orthonormality properties:

$$
\vec{V}_{(m)} \cdot \vec{V}_{(n)}=-\delta_{m n}, \quad \vec{W}_{(i)} \cdot \vec{W}_{(j)}=\delta_{i j}, \quad \vec{V}_{(m)} \cdot \vec{W}_{(i)}=0
$$

where the inner product is defined using the metric $\eta_{I J}$. A collection of vectors $\vec{V}_{(m)}$ and $\vec{W}_{(i)}$ satisfying the above orthogonality relations defines an element of $O(p, q)$.

If the mass matrix $m_{I J}$ is block-diagonal, i.e, $m_{i m}=0$, then we can always bring it to a diagonal form using matrices in $O(p) \times O(q) \subset O(p, q)$. Therefore in order for $m_{I J}$ to be diagonalisable, it is sufficient to check whether it can be brought into a block-diagonal form. There exists an element of $O(p, q)$ which achieves this if and only if we can find a collection of vectors $\vec{V}_{(m)}$ and $\vec{W}_{(i)}$ such that

$$
V_{(m)}^{I} W_{(i)}^{J} m_{I J}=0 \quad \forall m, i
$$

These then are the diagonalisability conditions. If they are satisfied the theory breaks up into decoupled free fields, but if not then it can exhibit more interesting behaviour.

\subsection{Necessary and sufficient conditions: two-field case}

Let us first look at a simple example for which $p=q=1$. We are working in a basis where the kinetic term has already been diagonalised and scaled, so $k_{I J}=(-1,1)$. The most general vectors $\vec{V}$ and $\vec{W}$ satisfying the conditions above are:

$$
\vec{V}=(\sinh \eta, \cosh \eta), \quad \vec{W}= \pm(\cosh \eta, \sinh \eta)
$$

Parametrising:

$$
m_{I J}=\left(\begin{array}{ll}
a & b \\
b & c
\end{array}\right)
$$

we easily find that Eq. (2.23) reduces to:

$$
\frac{a+c}{2 b}=\operatorname{coth} 2 \eta
$$

from which the condition for diagonalisability follows:

$$
2|b|<|a+c|
$$


The eigenvectors and eigenvalues of $m_{I J}$ are not invariant under Lorentz transformations. Hence it is convenient to reformulate the above condition in terms of the (nonsymmetric) matrix $(\eta m)_{J}^{I}=\eta^{I K} m_{K J}$ :

$$
(\eta m)_{J}^{I}=\left(\begin{array}{cc}
-a & -b \\
b & c
\end{array}\right)
$$

Since it has one upper and one lower index, this matrix can be thought of as a linear transformation and one can ask for its eigenvectors and eigenvalues. These have been classified (in $3+1$ dimensions) in works on general relativity, for example Ref. [16]. In $1+1$ dimensions there are precisely three possibilities:

\section{$\underline{\text { Eigenvalues }}$}

(i) Two distinct, real

(ii) Two coincident

(iii) Complex-conjugate pair

\section{Eigenvectors}

Two distinct, real (one spacelike, one timelike)

One

Complex-conjugate pair

Case (i) allows us to make an $S O(1,1)$ matrix:

$$
\Lambda=\left(\begin{array}{ll}
\vec{v}_{t} & \vec{v}_{s}
\end{array}\right)
$$

where $\vec{v}_{t}, \vec{v}_{s}$ are the orthonormalised eigenvectors, the first one timelike and the second spacelike. Clearly $\Lambda$ diagonalises $\eta m$ by a similarity transformation:

$$
\Lambda^{-1} \eta m \Lambda=\eta m_{\text {diag }}
$$

where we have labelled the diagonal matrix as $\eta m_{\text {diag. }}$. Noting that $\Lambda^{-1}=\eta \Lambda^{T} \eta$, we see that:

$$
\Lambda^{T} m \Lambda=m_{\text {diag }}
$$

as desired. Clearly $\vec{v}_{t}, \vec{v}_{s}$ are just the same as $\vec{V}, \vec{W}$. The other cases do not permit diagonalisation of $\eta m$ and thereby of $m$.

It is easy to check that the three cases above correspond to three sets of values for the discriminant of the eigenvalue equation for $\eta m$, namely $\Delta=(a+c)^{2}-4 b^{2}$ :

$$
\begin{aligned}
& \text { (i) } \Delta>0 \rightarrow 2|b|<|a+c| \\
& \text { (ii) } \Delta=0 \rightarrow 2|b|=|a+c| \\
& \text { (iii) } \Delta<0 \rightarrow 2|b|>|a+c|
\end{aligned}
$$

Since case (i) admits diagonalisation of the theory, it is trivial. We therefore study the system for cases (ii) and (iii). Labelling the two vector fields as $A^{(1)}, A^{(2)}$, the Lagrangian 
is:

$$
\begin{gathered}
\mathcal{L}_{1}=-\frac{1}{2} A^{(1)} \wedge d A^{(1)}+\frac{1}{2} A^{(2)} \wedge d A^{(2)}+\frac{1}{2} a A^{(1)} \wedge^{*} A^{(1)} \\
+b A^{(1)} \wedge^{*} A^{(2)}+\frac{1}{2} c A^{(2)} \wedge^{*} A^{(2)}
\end{gathered}
$$

Note that this Lagrangian is parity conserving, where the parity operation is taken as a reflection of space together with the interchange $A^{(1)} \leftrightarrow A^{(2)}$.

We start with case (ii), namely $|a+c|=2|b|$. As an example choose $a=c=\frac{m}{2}, b=-\frac{m}{2}$, so the mass matrix is:

$$
m_{I J}=\frac{m}{2}\left(\begin{array}{cc}
1 & -1 \\
-1 & 1
\end{array}\right)
$$

Then:

$$
\mathcal{L}_{1}=-\frac{1}{2} A^{(1)} \wedge d A^{(1)}+\frac{1}{2} A^{(2)} \wedge d A^{(2)}+\frac{1}{4} m\left(A^{(1)}-A^{(2)}\right) \wedge^{*}\left(A^{(1)}-A^{(2)}\right)
$$

Since the Chern-Simons and mass terms are not simultaneously diagonalisable, it is not immediately apparent how to deduce the spectrum of this theory. However, a field redefinition in terms of even and odd parity eigenstates:

$$
\begin{aligned}
& C=\frac{1}{\sqrt{2}}\left(A^{(2)}+A^{(1)}\right) \\
& B=\frac{1}{\sqrt{2}}\left(A^{(2)}-A^{(1)}\right)
\end{aligned}
$$

casts the Lagrangian into the more useful form:

$$
\mathcal{L}_{1}=B \wedge d C+\frac{1}{2} m B \wedge{ }^{*} B
$$

Now one of the fields, namely $B$, is algebraic. The equations of motion are:

$$
{ }^{*} d C=m B, \quad d B=0
$$

and the first equation can be used to eliminate $B$. Inserting this back, the Lagrangian reduces to:

$$
\mathcal{L}_{2}=\frac{1}{2 m} d C \wedge^{*} d C
$$

We see that while $\mathcal{L}_{1}$ has the form of a generalised two-field self-dual theory, $\mathcal{L}_{2}$ is instead a massless Maxwell Lagrangian. In theories where the mass term of Eq. (2.36) arises from a Higgs mechanism, what happens physically is that the single degree of freedom of a real Higgs scalar gets traded for the single degree of freedom of a massless vector.

Instead of integrating out $B$, an equivalent way to understand Eq. (2.36) is to define the new 1-form field $B^{\prime}$ :

$$
B^{\prime}=B-\frac{1}{m} * d C
$$


in terms of which the action becomes:

$$
\mathcal{L}_{2}^{\prime}=\frac{1}{2 m} d C \wedge^{*} d C+\frac{1}{2} m B \wedge^{*} B
$$

and we have a free Maxwell field plus a decoupled auxiliary field.

The above example is precisely the free-field reduction of the one in which the NHM was originally discovered [ब]. Here we have derived it from a different point of view: by constructing the simplest Chern-Simons-mass theory where the Chern-Simons and mass terms cannot be simultaneously diagonalised.

To study the more general version of case (ii), as well as case (iii), we continue to work in the basis of definite-parity fields where the coefficient of the Chern-Simons term is:

$$
k_{I J}=\left(\begin{array}{cc}
0 & 1 \\
1 & 0
\end{array}\right)
$$

In this basis the general mass matrix Eq. (2.25) becomes:

$$
m_{I J}=\frac{1}{2}\left(\begin{array}{cc}
a+c-2 b & -a+c \\
-a+c & a+c+2 b
\end{array}\right)
$$

The Lagrangian is then:

$$
\mathcal{L}=B \wedge d C+\frac{1}{4}(a+c-2 b) B \wedge^{*} B+\frac{1}{2}(c-a) B \wedge^{*} C+\frac{1}{4}(a+c+2 b) C \wedge^{*} C
$$

If $a+c \neq 2 b$ then the equations of motion can be solved for $B$. Inserting this solution back into the action, we find:

$$
\mathcal{L}=\frac{1}{a+c-2 b}\left(d C \wedge^{*} d C+(a-c) C \wedge d C+\left(a c-b^{2}\right) C \wedge^{*} C\right)
$$

It is convenient to change the normalisation of $C$ at this point so that the coefficient $\frac{1}{a+c-2 b}$ of the Lagrangian becomes $\frac{1}{2}$. Then comparing with Eq. (2.10), we have:

$$
w=1, \quad x=c-a, \quad y=a c-b^{2}
$$

It follows from Eq. 2.11) that the spectrum of this theory generically contains a pair of states of masses:

$$
\frac{1}{2}\left(c-a \pm \sqrt{(a+c)^{2}-4 b^{2}}\right)
$$

As already noted, the spin of the state is given by the sign of the mass. For $a=c$ the theory is parity-conserving since the Chern-Simons term drops out, and there are two degenerate massive states of spin \pm 1 as required by parity.

The theory above has a massless propagating gauge field if and only if $x=y=0$. This means we must have $a=c= \pm b$. The positive sign is ruled out because we eliminated 
the field $B$ on the assumption that $a+c \neq 2 b$ (however there is no loss of generality, since if $a+c=2 b$ then we would have eliminated the field $C$ instead and then the negative sign would have been ruled out). In conclusion, we have shown that for two fields, the purely Chern-Simons-mass action of Eq. (2.12) with a Lorentzian-signature Chern-Simons term has a propagating massless mode if and only if $a=c=-b$, in other words precisely the mass matrix Eq. 2.34 that we used as an example of the NHM. This establishes that there is a unique case with two fields. While we had already seen that the discriminant $\Delta=(a+c)^{2}-4 b^{2}$ should be non-positive to ensure non-diagonalisability of the Lagrangian, we now have a sufficient condition for NHM that requires both $\Delta=0$ and $a=c$.

There are other interesting cases. Whenever $a c=b^{2}$, the system has only a single massive excitation. This can be seen directly from the Lagrangian where $B$ has been integrated out, namely Eq. (2.44), in which the explicit mass term drops out leaving a topologically massive theory whose gauge invariance decouples the second excitation. Another special case is when $|a+c|= \pm 2|b|$ but $a \neq c$. In this case one finds two massive states of the same mass and spin. This may be thought of as "maximal parity violation" and represents another of the interesting situations arising from non-diagonalisability of the action. While the same spectrum can also be obtained by just taking a pair of decoupled Chern-Simonsmass actions, the origin of parity violation is different: in the latter case it arises from the Chern-Simons terms while in the former case it comes from the mass terms. This difference will be relevant after introducing interactions.

Finally, case (iii) in the notation of Eq. (2.32) corresponds to a discriminant $\Delta<0$. In this case the spectrum consists of a conjugate pair of complex masses of spins \pm 1 . We reserve judgement on whether such a theory is necessarily inconsistent, since interactions might conceivably render it consistent. Note that the fields cannot be redefined to make the masses purely real or imaginary (tachyonic) because those states would not then be spin eigenstates. (In the parity conserving case $c=a$, the masses do become purely imaginary.) Therefore the complex-mass case is one of the interesting features arising in the spectrum of two-field models. We also see that the novel Higgs mechanism occurs on the boundary between a pair of topologically massive Chern-Simons theories and a Maxwell theory with a complex mass.

\section{General number of fields}

\subsection{Non-diagonalisability: a necessary condition}

There are several different ways to describe the solution to the conditions for the novel Higgs mechanism in the general situation with three or more gauge fields. One of these, 
which follows from considerations that we analysed in the previous subsection, is the following. Consider the matrix $m_{J}^{I}=\eta^{I K} m_{K J}$. If this matrix has $p$ distinct real timelike eigenvectors and $q$ distinct real spacelike eigenvectors then it can be diagonalised by a similarity transformation involving an $S O(p, q)$ matrix, otherwise not. If it is not diagonalisable then we may look for interesting phenomena including generalisations of the NHM.

The nature of possible eigenvectors of such a matrix in a space of Lorentzian signature is reviewed in [16] (for the case of $3+1$ dimensions) using notation due to Segré and Plebański. The case where the matrix possesses a maximal set of nondegenerate eigenvalues and corresponding distinct eigenvectors is referred to as algebraically general. In Segré notation, an algebraically general tensor has the label $(11 \cdots 1,11 \cdots 1)$ with $p$ entries before the comma representing timelike eigenvectors and $q$ entries after the comma representing spacelike eigenvalues. All other cases are said to be algebraically special. These then are the cases for which one has a non-diagonalisable mass matrix. The novel Higgs mechanism and any other interesting phenomena can therefore arise only for the algebraically special case.

There is another way of stating the general diagonalisability condition Ref. 17, 18. that will be more useful for us. These works contain a theorem on the possibility of simultaneously diagonalising a pair of quadratic forms $(A, B)$. In Section 12.12 of Ref. 17 it is proved that quadratic forms $A$ and $B$ in more than two variables can be simultaneously diagonalised by a linear transformation if they have no common zeros along the diagonal in any basis $^{2}$.

For us the two quadratic forms are $k_{I J}$ and $m_{I J}$. It is most convenient to choose a maximally off-diagonal basis for the former. If we have $p$ timelike and $q$ spacelike directions with $p<q$ (the analysis is similar for $p \geq q$ ) we can bring $k_{I J}$ to the form:

$$
k_{I J}=\left(\begin{array}{ccc}
0 & \mathbb{I}_{p} & 0 \\
\mathbb{I}_{p} & 0 & 0 \\
0 & 0 & \mathbb{I}_{q-p}
\end{array}\right)
$$

From now on we always work in this basis. Then applying the theorem quoted above, $m_{I J}$ will be diagonalisable it does not have any zeroes on the diagonal in this basis. This then is the condition under which the theory splits into a sum of decoupled theories of the form of Eq. (2.10).

\footnotetext{
${ }^{2}$ Notice that we have already encountered the exception to this theorem in the two-variable case of the previous section. The non-diagonalisable case $\Delta<0$ discussed there includes a mass matrix with $a=0$ or $c=0$, in a basis where the kinetic matrix is $\operatorname{diag}(-1,1)$. Thus the two matrices do not have common zeroes along the diagonal but nevertheless cannot be diagonalised together.
} 
One expects that all other cases, namely those where $m_{I J}$ has at least one zero on the diagonal in its first $2 p \times 2 p$ block, must represent something more exotic than a collection of decoupled fields. However that does not mean they all involve massless propagating fields. We have only found a necessary condition for this, and in the next section we will look for sufficient conditions.

\subsection{Sufficient conditions: three field case}

Let us now consider the case with three fields and a kinetic matrix:

$$
\eta_{I J}=\left(\begin{array}{lll}
0 & 1 & 0 \\
1 & 0 & 0 \\
0 & 0 & 1
\end{array}\right)
$$

The most general Chern-Simons-mass theory with this content has the Lagrangian:

$$
\begin{aligned}
\mathcal{L}= & B \wedge d C+\frac{1}{2} D \wedge d D+\frac{1}{2} \alpha B \wedge{ }^{*} B+\frac{1}{2} \beta C \wedge{ }^{*} C+\frac{1}{2} \gamma D \wedge^{*} D \\
& +\mu B \wedge{ }^{*} C+\nu C \wedge{ }^{*} D+\rho D \wedge{ }^{*} B
\end{aligned}
$$

The equation of motion of $B$ is:

$$
d C+\alpha^{*} B+\mu^{*} C+\rho^{*} D=0
$$

Solving for $B$ and inserting back in the Lagrangian, we find:

$$
\begin{aligned}
\mathcal{L}= & \frac{1}{2 \alpha} d C \wedge^{*} d C-\frac{\mu}{\alpha} C \wedge d C+\frac{1}{2}\left(\beta-\frac{\mu^{2}}{\alpha}\right) C \wedge{ }^{*} C-\frac{\rho}{\alpha} D \wedge d C \\
& +\left(\nu-\frac{\mu \rho}{\alpha}\right) C \wedge^{*} D+\frac{1}{2} D \wedge d D+\frac{1}{2}\left(\gamma-\frac{\rho^{2}}{\alpha}\right) D \wedge^{*} D
\end{aligned}
$$

Thus we have a Maxwell coefficient matrix $Y_{I J}$, a Chern-Simons matrix $k_{I J}$ and a mass matrix $m_{I J}$ given by:

$$
Y_{I J}=\left(\begin{array}{cc}
\frac{1}{\alpha} & 0 \\
0 & 0
\end{array}\right), \quad k_{I J}=\left(\begin{array}{cc}
-\frac{2 \mu}{\alpha} & -\frac{\rho}{\alpha} \\
-\frac{\rho}{\alpha} & 1
\end{array}\right), \quad m_{I J}=\left(\begin{array}{cc}
\beta-\frac{\mu^{2}}{\alpha} & \nu-\frac{\mu \rho}{\alpha} \\
\nu-\frac{\mu \rho}{\alpha} & \gamma-\frac{\rho^{2}}{\alpha}
\end{array}\right)
$$

Evidently the NHM, leading to a single massless propagating excitation, arises only if $\beta=\gamma=\mu=\nu=\rho$ so there is no new example with three fields relative to the case already analysed for two fields. However the spectrum is in general far more complicated. The two-field case was previously discussed in Sec.2.3, but with only Chern-Simons and mass terms. Now that a Maxwell term is also present, it is impossible to diagonalise all three terms simultaneously in general. Since the Maxwell term goes away in the far infrared, the analysis for an interacting theory with the above quadratic terms will follow the analysis of 
Sec.2.3. For this we need to inspect Eq. (3.6) above which tells us that the Chern-Simons term has indefinite signature if:

$$
\operatorname{det} k_{I J}=-\frac{2 \mu}{\alpha}-\frac{\rho^{2}}{\alpha^{2}}<0
$$

Therefore this is the case for which, at least in the infrared, the Lagrangian cannot be diagonalised but may be analysed following the procedure outlined above.

\subsection{General case}

The sufficient conditions for NHM in the multi-field case, at least for $p=q$ (equal number of positive and negative eigenvalues for the Chern-Simons coefficient) can be found by repeating the procedure for the two-field case. We work in the basis where the ChernSimons coefficient $k_{I J}$ is given by Eq. (3.1) and divide the $A^{(I)}, I=1,2, \cdots, 2 p$ into two sets:

$$
\begin{aligned}
A^{i} & =B^{i}, i=1,2, \cdots, p \\
A^{p+i} & =C^{i}, i=1,2, \cdots, p
\end{aligned}
$$

Then the free Chern-Simons-mass Lagrangian takes the form:

$$
\mathcal{L}=B^{i} \wedge d C^{i}+\frac{1}{2} \alpha_{i j} B^{i} \wedge{ }^{*} B^{j}+\beta_{i j} B^{i} \wedge{ }^{*} C^{j}+\frac{1}{2} \gamma_{i j} C^{i} \wedge{ }^{*} C^{j}
$$

and the corresponding equations of motion are:

$$
\begin{aligned}
& d C^{i}+\alpha_{i j}{ }^{*} B^{j}+\beta_{i j}{ }^{*} C^{j}=0 \\
& d B^{i}+\beta_{i j}{ }^{*} B^{j}+\gamma_{i j}{ }^{*} C^{j}=0
\end{aligned}
$$

Now suppose the matrix $\alpha_{i j}$ is invertible. In that case we can solve the first equation for $B^{i}$ and insert this back into the original Lagrangian to get:

$$
\mathcal{L}=\frac{1}{2} \alpha_{i j}^{-1} d C^{i} \wedge^{*} d C^{j}-\left(\alpha^{-1} \beta\right)_{i j} C^{i} \wedge d C^{j}+\frac{1}{2}\left(\gamma-\beta \alpha^{-1} \beta\right)_{i j} C^{i} \wedge^{*} C^{j}
$$

The Chern-Simons term vanishes for every zero eigenvector of $\beta$. Moreover if such an eigenvector is a simultaneous zero eigenvector of $\gamma$ then the mass term also vanishes. We conclude that there is one massless propagating vector field for every simultaneous zero eigenvector of the matrices $\beta_{i j}$ and $\gamma_{i j}$, under the condition that $\alpha_{i j}$ is invertible. As in the two-field case, the roles of $\alpha_{i j}$ and $\gamma_{i j}$ can be interchanged. If $\alpha_{i j}$ is not invertible, as in a case we will encounter in the following subsection, then we need that it is nonzero on the common zero eigenvector of $\beta, \gamma$. 


\subsection{Higgs fields}

So far we have been working with generic mass matrices, not specifying precisely how they arise via a Higgs mechanism. Here we want to ask which kinds of charged fields give rise to the different types of mass matrices discussed above. Let us work with an even number $2 p$ of gauge fields. Since we are still working in the Abelian theory, each Higgs field is a scalar $\phi_{\vec{q}}$ carrying a set of charges under these gauge fields. To analyse the physics one needs to fix the basis for the gauge fields. It is easiest to choose this as in the previous subsection, i.e. divide them into two sets $B_{i}, C_{i}, i=1,2 \ldots, p$.

Next we choose the charges of a particular Higgs field to be $\vec{q}=\left(q_{1}, q_{2}, \cdots q_{p} ; r_{1}, r_{2} \cdots, r_{p}\right)$ where the $q_{i}$ are charges under $B_{i}$ and the $r_{i}$ are charges under $C_{i}$. The kinetic term for such a scalar is:

$$
\left|\left(\partial_{\mu}-i \sum_{i=1}^{p} q_{i} B_{\mu}^{i}-i \sum_{i=1}^{p} r_{i} C_{\mu}^{i}\right) \phi_{\vec{q}}\right|^{2}
$$

If $\phi_{\vec{q}}$ acquires a (complex) vev $\left\langle\phi_{\vec{q}}\right\rangle=v_{\vec{q}}$, one gets a mass matrix:

$$
m_{I J}=2\left|v_{\vec{q}}\right|^{2}\left(\begin{array}{cc}
q_{i} q_{j} & q_{i} r_{j} \\
r_{i} q_{j} & r_{i} r_{j}
\end{array}\right)
$$

We can identify the matrices $\alpha_{i j}, \beta_{i j}, \gamma_{i j}$ of the previous subsection with $q_{i} q_{j}, q_{i} r_{j}, r_{i} r_{j}$ respectively (upto an overall proportionality constant). Now any vector $v_{j}$ orthogonal to $r_{j}$, i.e. satisfying $r_{j} v_{j}=0$, will be a common zero eigenvector of the matrices $\beta, \gamma$ and, according to the analysis of the previous subsection, can give rise to a massless propagating field. However $q_{i} q_{j}$ is also singular, with only one nonzero eigenvector $q_{j}$ itself. Therefore to end up with a massless propagating field requires $q_{j} v_{j} \neq 0$.

As a by-now familiar example, take $p=1, N_{f}=1$, and let the single scalar carry charges $(q ; r)$. The mass matrix is then:

$$
m_{I J}=2\left|v_{\vec{q}}\right|^{2}\left(\begin{array}{cc}
q^{2} & q r \\
q r & r^{2}
\end{array}\right)
$$

With $q$ or $r$ vanishing, this gives us $a=c=|b|$ in the previous notation and one has NHM. Thus we have rederived the well-known result [4, 10] that with two gauge fields $A^{1}, A^{2}$ (in a diagonal basis for the Chern-Simons term) and one scalar, the latter must be equally or oppositely charged under both ("bi-fundamental", in the non-Abelian case) to give rise to the novel Higgs mechanism. Moreover, this is seen to be essentially unique. In the generic case with both $q, r \neq 0$, one easily sees that $|a+c|>2|b|$ so the action is diagonalisable and there are no novel features. 
With a number of independent Higgs fields $\phi_{\vec{q}^{A}}, A=1,2, \cdots, N_{f}$ we get the mass matrix:

$$
m_{I J}=2 \sum_{i=1}^{N_{f}}\left|v_{\vec{q}^{A}}\right|^{2}\left(\begin{array}{cc}
q_{i}^{A} q_{j}^{A} & q_{i}^{A} r_{j}^{A} \\
r_{i}^{A} q_{j}^{A} & r_{i}^{A} r_{j}^{A}
\end{array}\right)
$$

In this case one can achieve the maximal number of massless propagating fields, namely $p$, by simply taking $N_{f}=p$ scalars each with $q_{i} \neq 0$ for one $i$ and $r_{i}=0$ for all $i$. This amounts to a straightforward set of copies of the two-field bi-fundamental case, but then one should not expect to find anything more than that at the Abelian level. Things can become more complicated when there are non-Abelian interactions.

\section{Non-abelian case}

The non-Abelian Chern-Simons system with multiple fields presents some new features that influence the NHM. One well-known feature is that the coefficient of the Chern-Simons term is quantised for compact gauge groups and we cannot ignore it or scale it to unity as we did in the preceding sections.

\subsection{Difference Chern-Simons}

Let us start with the simplest example, a $\mathcal{G} \times \mathcal{G}$ theory with level $k$ for both the gauge groups. We write the difference Chern-Simons action as:

$$
L_{C S}=\frac{k}{4 \pi} \operatorname{tr}\left(A \wedge d A+\frac{2}{3} A \wedge A \wedge A-\tilde{A} \wedge d \tilde{A}-\frac{2}{3} \tilde{A} \wedge \tilde{A} \wedge \tilde{A}\right)
$$

where $A=A^{a} T^{a}$ and $\operatorname{tr} T^{a} T^{b}=-\frac{1}{2} \delta^{a b}$. For compact $\mathcal{G}, k$ is required to be an integer in order to have gauge invariance under large gauge transformations [3].

Comparing with our discussion above, this corresponds to the basis in which the coefficient matrix $\eta_{I J}=\operatorname{diag}(-1,1)$. In this basis the non-Abelian gauge invariance is the normal one:

$$
\delta A=d \Lambda+[A, \Lambda], \quad \delta \tilde{A}=d \tilde{\Lambda}+[\tilde{A}, \tilde{\Lambda}]
$$

The second basis, in which $\eta_{I J}$ is purely off-diagonal, is obtained as before by taking the linear combinations

$$
B=\frac{1}{2}(A-\tilde{A}), \quad C=\frac{1}{2}(A+\tilde{A}), \quad F^{(C)}=d C+C \wedge C
$$

(the fields $B$ and $C$ thereby correspond to those in the Abelian case, but normalised slightly differently to avoid ugly factors of $\sqrt{2}$ in the Lagrangian). In these variables, the Lagrangian becomes:

$$
L_{C S}=\frac{k}{\pi} \operatorname{tr}\left(B \wedge F^{(C)}+\frac{1}{3} B \wedge B \wedge B\right)
$$


and the gauge transformations take the somewhat non-standard form:

$$
\begin{aligned}
& \delta B=d \Lambda_{B}+\left[C, \Lambda_{B}\right]+\left[B, \Lambda_{C}\right] \\
& \delta C=d \Lambda_{C}+\left[C, \Lambda_{C}\right]+\left[B, \Lambda_{B}\right]
\end{aligned}
$$

The equations of motion in the $A, \tilde{A}$ basis are just the equations for two independent flat connections:

$$
F=\tilde{F}=0
$$

However in the $B, C$ basis they look like:

$$
\begin{array}{r}
F^{(C)}+B \wedge B=0 \\
D^{(C)} B \equiv d B+[C, B]=0
\end{array}
$$

Now we would like to give a mass via the Higgs mechanism. The structure of the mass matrix arises via the choice of representation of the group $\mathcal{G} \times \mathcal{G}$ under which the Higgs field transforms. The canonical choice is the bi-fundamental, for example the $(N, \bar{N}$ of $S U(N)$ :

$$
\delta \Phi=-\Lambda \Phi+\Phi \tilde{\Lambda}
$$

In this case the covariant derivative is:

$$
D_{\mu} \Phi=\partial_{\mu} \Phi+A_{\mu} \Phi-\Phi \tilde{A}_{\mu}
$$

For convenience we will normalise the scalar kinetic term as:

$$
\frac{k}{4 \pi} \operatorname{tr}\left(D_{\mu} \Phi^{\dagger} D^{\mu} \Phi\right)
$$

where this trace is, formally, unrelated to that in the gauge field action - here it just sums over two pairs of repeated indices in the fundamental representation, one pair being associated to each gauge group of $\mathcal{G} \times \mathcal{G}$. This kinetic term gives rise to the interaction:

$$
\frac{k}{4 \pi} \operatorname{tr}\left|A_{\mu} \Phi-\Phi \tilde{A}_{\mu}\right|^{2}
$$

With a Higgs vev proportional to the identity: $\langle\Phi\rangle=v \mathbb{I}$, the mass term is equal to

$$
\frac{k}{4 \pi} v^{2} \operatorname{tr}\left(A_{\mu}-\tilde{A}_{\mu}\right)^{2}
$$

where now the trace is over the Lie algebra of $\mathcal{G}$ after identifying the two factors in $\mathcal{G} \times \mathcal{G}$. We see that the mass matrix has the form:

$$
m_{I J} \sim\left(\begin{array}{rr}
1 & -1 \\
-1 & 1
\end{array}\right)
$$


which we have encountered many times over as the basic example of NHM.

This time, however, one has to keep track of the cubic terms in $B$. With the above vev, one has the Lagrangian:

$$
\mathcal{L}=\frac{k}{\pi} \operatorname{tr}\left(B \wedge F^{(C)}+\frac{1}{3} B \wedge B \wedge B-v^{2} B \wedge^{*} B\right)
$$

From this follows the equation of motion for $B$ :

$$
F^{(C)}+B \wedge B-2 v^{2 *} B=0
$$

The quadratic term in $B$ now makes it impossible, unlike in the Abelian case, to simply solve for $B$ and eliminate it. The best we can do is solve recursively, to get:

$$
\begin{aligned}
B & =-\frac{1}{2 v^{2}}{ }^{*} F^{(C)}-{\frac{1}{2 v^{2}}}^{*}(B \wedge B) \\
& =-\frac{1}{2 v^{2}}{ }^{*} F^{(C)}-{\frac{1}{8 v^{6}}}^{*}\left({ }^{*} F^{(C)} \wedge{ }^{*} F^{(C)}\right)+\cdots
\end{aligned}
$$

The terms in $\cdots$ above contain all powers of $F^{(C)}$, appearing in combinations like:

$$
{ }^{*}\left({ }^{*} F^{(C)} \wedge{ }^{*}\left({ }^{*} F^{(C)} \wedge \cdots{ }^{*}\left(F^{(C)} \wedge{ }^{*} F^{(C)}\right)\right)\right)
$$

These are made by wedging the 1-form dual to $F^{(C)}$ with itself, then dualising the result back to a 1-form and repeating indefinitely. This nonlinear combination of field strengths appears to be unique to $(2+1) \mathrm{d}$. Moreover, the orders in this expansion are counted by the parameter $\frac{1}{v^{2}}$.

We may now insert Eq. (4.16) back into the Lagrangian of Eq. (4.14) to find:

$$
\mathcal{L}=\frac{k}{\pi}\left(-\frac{1}{4 v^{2}} F^{(C)} \wedge{ }^{*} F^{(C)}-\frac{1}{24 v^{6}}{ }^{*} F^{(C)} \wedge^{*} F^{(C)} \wedge{ }^{*} F^{(C)}+\cdots\right)
$$

We see that taking $v \rightarrow \infty$ allows us to ignore the higher-order terms in $F^{(C)}$. However, in the quadratic term, $v^{2}$ plays the role of the Yang-Mills coupling constant $\llbracket$. Therefore in the same limit that decouples the higher-order terms, the Yang-Mills term becomes very strongly coupled. This can be avoided by simultaneously scaling $k \rightarrow \infty, v \rightarrow \infty$ keeping $\frac{k}{v^{2}}$ fixed[19]. In this limit the higher-order terms do drop out, but the Yang-Mills coupling $\frac{v}{\sqrt{k}}$ remains finite and can be chosen arbitrarily.

One can take the Higgs field in a slightly different representation that is equivalent to the previous one in the Abelian case but differs in the non-Abelian case. This is the bi-fundamental $(N, N)$ rather than $(N, \bar{N})^{3}$. In this case the gauge transformation is:

$$
\delta \Phi=-\Lambda \Phi-\Phi \tilde{\Lambda}
$$

\footnotetext{
${ }^{3}$ This representation arises in the fermionic sector of certain orientifold field theories, as in Ref. [20].
} 
and the covariant derivative is:

$$
D_{\mu} \Phi=\partial_{\mu} \Phi+A_{\mu} \Phi+\Phi \tilde{A}_{\mu}
$$

This time the vev $\langle\Phi\rangle=v \mathbb{I}$ gives rise to a mass matrix:

$$
m_{I J} \sim\left(\begin{array}{ll}
1 & 1 \\
1 & 1
\end{array}\right)
$$

which at the Abelian level has the same properties as Eq. (4.13) above and gives rise to the NHM, with the difference that the field $C \sim A+\tilde{A}$ is eliminated and the field $B \sim A-\tilde{A}$ becomes propagating. However in the non-Abelian case the cubic terms are different. The Lagrangian after Higgsing is:

$$
\mathcal{L}=\frac{k}{\pi} \operatorname{tr}\left(B \wedge F^{(C)}+\frac{1}{3} B \wedge B \wedge B-v^{2} C \wedge^{*} C\right)
$$

We need to integrate out $C$, so the first term has to be rewritten:

$$
\begin{aligned}
B \wedge F^{(C)} & =B \wedge d C+B \wedge C \wedge C \\
& =C \wedge d B+B \wedge C \wedge C
\end{aligned}
$$

where we have performed integration by parts on the kinetic term. The equation of motion for $C$ is:

$$
d B+2 B \wedge C-2 v^{2}{ }^{*} C=0
$$

which gives:

$$
\begin{aligned}
C & =-\frac{1}{2 v^{2}} * d B-{\frac{1}{v^{2}}}^{*}(B \wedge C) \\
& =-\frac{1}{2 v^{2}} * d B+\frac{1}{2 v^{4}} *\left(B \wedge^{*} d B\right)+\cdots
\end{aligned}
$$

This time the higher-order terms are made up of combinations like:

$$
*\left(B \wedge{ }^{*}\left(B \wedge \cdots{ }^{*}\left(B \wedge{ }^{*} d B\right)\right)\right)
$$

which should be contrasted with Eq. 4.17).

It is manifest from the construction of Eq. (4.17) and Eq. (4.26) that these nonlinear terms are gauge invariant (after taking a trace) under the transformations in Eq. (4.5). These (perhaps novel) gauge-invariant combinations are building blocks that may have interesting applications in the study of M-theory membrane actions.

One can consider yet other representations for the Higgs field(s). But as in the Abelian case, it is clear that fields which transform only under one or other gauge group give uninteresting decoupled gauge symmetries. 


\section{$4.2 B \wedge F$ theory}

There is a different non-Abelian generalisation of the Abelian difference Chern-Simons theory. Instead of writing a difference of two non-Abelian Chern-Simons terms, we start directly with the action:

$$
2 \operatorname{tr} B \wedge F^{(C)}
$$

with gauge invariances:

$$
\begin{aligned}
\delta B & =d \Lambda_{B}+\left[C, \Lambda_{B}\right]+\left[B, \Lambda_{C}\right] \\
\delta C & =d \Lambda_{C}+\left[C, \Lambda_{C}\right]
\end{aligned}
$$

At the Abelian level this is still the difference of two Chern-Simons actions. However the absence of the last term in $\delta C$ as compared with Eq. (4.5) means the non-Abelian gauge invariance is different. Introducing a suitable scalar field one can get a mass term:

$$
v^{2} \operatorname{tr} B_{\mu} B^{\mu}
$$

Because of the absence of a $B^{3}$ term, this time the end-point of the NHM is the massless Yang-Mills theory without any corrections. This means the theory above can be thought of as a reformulation of $(2+1)$ d Yang-Mills theory, a point which was stressed in the M-theory context in Ref. 21].

The theory in Eq. (4.27) together with the mass term in Eq. 4.29) can be obtained by starting with Eq. (4.14), performing the re-scaling:

$$
B \rightarrow \lambda^{-1} B, \quad k \rightarrow \lambda k, \quad v \rightarrow \sqrt{\lambda} v
$$

and then taking the limit $\lambda \rightarrow \infty$. In this sense it is just a limiting case of difference Chern-Simons theory. Note that the final Yang-Mills coupling $g_{Y M}{ }^{2} \sim v^{2} / k$ remains finite in this limit. It is not clear that $k$ should be quantised in the final theory, in fact it can be absorbed in a redefinition of $B$.

\section{Potential applications}

In this section I offer some speculative ideas about the possible occurrence of the NHM in the context of condensed-matter physics as well as gravity. Though there will be no definite conclusion in either case, it is tantalising that known field theories arising in both contexts quite naturally exhibit a structure of multiple gauge fields and a difference-Chern-Simons Lagrangian. Therefore it is plausible that, with more investigation, a physical setting for the NHM can be found in each case. 


\subsection{Condensed-matter systems}

Abelian Chern-Simons gauge fields play a key role in understanding phenomena like the fractional quantum Hall effect (see for example Refs. [0, 6, 7, 8]). In particular the ChernSimons term generates a change from Bose/Fermi to anyon statistics. Several gauge fields appear in the problem. To start with there is of course the electromagnetic field, which being massless has only a Maxwell term. Subsequently a "statistical gauge field" is introduced, having a Chern-Simons term with a suitable coefficient. In the treatment of Ref. [8] there are also several other gauge fields. A set of $m$ gauge fields is introduced so that the fermionic operators in the Lagrangian can be replaced by bosonic operators, one for each of $m$ Landau levels. Another set comes in upon dualising the Goldstone modes into gauge fields using scalar-gauge duality in $(2+1)$ d.

Let us briefly review this and see how structures similar to those we have been discussing in previous sections could arise. In units where $\hbar=c=e=1$, e being the electric charge quantum, a fractional Hall system in some definite Landau level is described by the Lagrangian (the electrons $\psi$ are nonrelativistic):

$$
\mathcal{L}=\psi^{\dagger} i\left(\partial_{0}-i A_{0}\right) \psi+\frac{1}{2 m} \psi^{\dagger}\left(\partial_{i}-i A_{i}\right)^{2} \psi-\frac{1}{4} F_{\mu \nu} F^{\mu \nu}
$$

where $A_{\mu}$ is the external electromagnetic field. The filling fraction is $\nu=\frac{2 \pi n}{B}$ where $n$ is the number of electrons per unit area and $B$ is the magnetic field, $B=d A$.

We now modify this Lagrangian by introducing a new Abelian gauge field $\alpha_{\mu}$ and modify the above Lagrangian to:

$$
\begin{aligned}
\mathcal{L}=\psi^{\dagger} i & \left(\partial_{0}-i\left(A_{0}+\alpha_{0}\right)\right) \psi+\frac{1}{2 m} \psi^{\dagger}\left(\partial_{i}-i\left(A_{i}+\alpha_{i}\right)\right)^{2} \psi \\
+ & \frac{1}{2} d A \wedge^{*} d A+\frac{1}{4 \pi p} \alpha \wedge d \alpha
\end{aligned}
$$

The equation of motion for $\alpha_{\mu}$ is:

$$
{ }^{*} d \alpha=-2 \pi p j
$$

where $j_{\mu}=\left(\psi^{\dagger} \psi, \psi^{\dagger} \vec{\nabla} \psi\right)$ is the current. It follows that the electrons effectively move in a reduced magnetic field $B_{\text {eff }}=B-2 \pi p n$. Thereby the filling fraction changes to:

$$
\nu \rightarrow \nu_{\text {eff }}=\frac{2 \pi n}{B_{\text {eff }}}=\frac{\nu}{1-p \nu}
$$

If the original filling fraction is of the form $\nu=\frac{m}{m p+1}$ with $m$ an integer, then we find $\nu_{\text {eff }}=m$. The quantity $p$ also contributes to the effective statistics of the electrons, which now have $p$ flux lines attached to them providing a phase of $e^{i \pi p}$. Therefore if we want to retain Fermi statistics, $p$ should be a (positive or negative) even integer. The net result is that we now have a system of fermions in the reduced magnetic field exhibiting the integer 
quantum Hall effect 22, 23. The field $\alpha$ is called the "statistical gauge field" for reasons which should be clear.

The next step in Ref.[8] is to replicate the above system over $m$ different Landau levels, with a Fermi field $\psi_{I}$ for each level. Then, introduce a new set of $m$ gauge fields $a_{I \mu}, I=1,2, \cdots, m$ with a suitable Chern-Simons term that converts each fermion field $\psi_{I}$ to a boson $\phi_{I}$. At this stage the Lagrangian is:

$$
\begin{gathered}
\mathcal{L}=\sum_{I=1}^{m}\left[\phi_{I}^{\dagger} i\left(\partial_{0}-i\left(A_{0}+\alpha_{0}+a_{I 0}\right)\right) \phi_{I}+\frac{1}{2 M} \phi_{I}^{\dagger}\left(\partial_{i}-i\left(A_{i}+\alpha_{i}+a_{I i}\right)\right)^{2} \phi_{I}\right. \\
\left.-V_{I}\left(\phi_{I}^{\dagger} \phi_{I}\right)+\frac{1}{4 \pi} a_{I} \wedge d a_{I}\right]+\frac{1}{2} d A \wedge^{*} d A+\frac{1}{4 \pi p} \alpha \wedge d \alpha
\end{gathered}
$$

where a potential $V_{I}\left(\phi_{I}^{\dagger} \phi_{I}\right)$ has been introduced to represent the interaction between electrons in the corresponding Landau level.

For simplicity let us set $m=1$. Ignoring the physical electromagnetic field for the moment, the gauge-field terms in the above Lagrangian are:

$$
\begin{aligned}
\mathcal{L}=\phi^{\dagger} i\left(\partial_{0}\right. & \left.-i\left(\alpha_{0}+a_{0}\right)\right) \phi+\frac{1}{2 M} \phi^{\dagger}\left(\partial_{i}-i\left(\alpha_{i}+a_{i}\right)\right)^{2} \phi \\
+\frac{1}{4 \pi} a & \wedge d a+\frac{1}{4 \pi p} \alpha \wedge d \alpha
\end{aligned}
$$

This looks something like the two-field system we analysed earlier, or rather a non-relativistic version of it. With a Higgs vev, the mass matrix will acquire the form:

$$
m_{I J} \sim\left(\begin{array}{ll}
1 & 1 \\
1 & 1
\end{array}\right)
$$

The physics now depends on the parameter $p$. If $p$ is positive then the mass term can be diagonalised, one eigenvalue is zero and the other nonzero, leading to one field remaining non-dynamical and the other being massive and propagating. If $p$ is negative then the Chern-Simons kinetic matrix has indefinite signature and the action is potentially non-diagonalisable. In this case, after scaling fields so that the kinetic matrix becomes $\operatorname{diag}(-1,1)$ the mass matrix takes the form:

$$
m_{I J} \sim\left(\begin{array}{cc}
1 & \sqrt{|p|} \\
\sqrt{|p|} & |p|
\end{array}\right)
$$

For any even $p$ this always satisfies the constraint in Eq. 2.27) and therefore the Lagrangian is again diagonalisable and leads to the same spectrum. This is satisfying because in the FQHE system the physics does not appear to depend significantly on the sign of the ChernSimons terms, which are introduced for the sake of statistics. 
It remains to consider $p=-1$, a case that was not allowed in the FQHE context. Here we indeed encounter the novel Higgs mechanism ${ }^{4}$ and the spectrum has a single propagating massless mode. However we need to examine whether the analysis is valid in the presence of the external gauge field $A$, which has a Maxwell kinetic term but mixes in the mass matrix with both the Chern-Simons fields. The resulting theory actually resembles the 3 -field case analysed in Section [3.2, with the relabelling $\left(B_{\mu}, C_{\mu}\right) \rightarrow\left(a_{\mu} \pm \alpha_{\mu}\right)$ and $D_{\mu} \rightarrow A_{\mu}$. With this, the 3-field mass matrix in Eq. (3.3) becomes:

$$
\left(\begin{array}{lll}
\alpha & \mu & \rho \\
\mu & \beta & \nu \\
\rho & \nu & \gamma
\end{array}\right) \rightarrow \sim v^{2}\left(\begin{array}{lll}
2 & 0 & 1 \\
0 & 0 & 0 \\
1 & 0 & 1
\end{array}\right)
$$

so $\mu=\nu=\beta=0$. After eliminating $B_{\mu}$, we find for the analogue of Eq. (3.6) the following matrices (here we have taken account of the fact that the field $A_{\mu}$ has a Maxwell rather than Chern-Simons kinetic term):

$$
Y_{I J}=\left(\begin{array}{cc}
\frac{1}{2 v^{2}} & 0 \\
0 & 1
\end{array}\right), \quad k_{I J}=\left(\begin{array}{cc}
0 & -\frac{1}{2} \\
-\frac{1}{2} & 0
\end{array}\right), \quad m_{I J}=v^{2}\left(\begin{array}{cc}
0 & 0 \\
0 & \frac{1}{2}
\end{array}\right)
$$

The above matrices are written in the basis of fields $\left(C_{\mu}, A_{\mu}\right)$. In the absence of the electromagnetic field $A_{\mu}$ we would retain only the top-left corner of these matrices and would find, as discussed above, that $C_{\mu}=a_{\mu}-\alpha_{\mu}$ is a massless propagating field. However we now see that the presence of $A_{\mu}$ complicates the theory considerably. In fact, elimination of $B_{\mu}$ has induced a Chern-Simons term for the electromagnetic field $A_{\mu}$ and the Chern-Simons kinetic matrix above is of Minkowski signature, so it cannot be diagonalised simultaneously with the mass matrix. Matters are complicated by the presence of Maxwell terms for both fields, but if it can be justified to ignore them at long distances then we would have a fresh novel Higgs mechanism leaving one combination of the electromagnetic field $A_{\mu}$ and $C_{\mu}=a_{\mu}-\alpha_{\mu}$ as a massless propagating field.

The purpose of this sub-section has not been to present and solve for a specific condensed-matter system exhibiting NHM (in particular, recall that the discussion above was carried out for $p=-1$ while $p$ is supposed to be even in the given systems). Instead it has been pointed out that the kind of theories discussed in the context of the quantum Hall effect, and more generally theories in which vortices and unusual statistics play an important role, do have multiple Chern-Simons terms and these can be mutually non-diagonalisable with the mass terms, making them plausible settings for the NHM. The study of what precise physical effect this induces, and in which specific many-body system, is left for future work.

\footnotetext{
${ }^{4}$ In the present context, perhaps it is better called the "novel Anderson-Higgs mechanism"...
} 


\section{$5.2(2+1) \mathrm{d}$ gravity}

It is known[24, 25] that in $2+1$ dimensions, gravity can be written as a difference-ChernSimons theory. One takes the variables to be the dreibein $e_{\mu}^{a}$ and $\omega_{\mu}^{a b}$ as is usual in the first-order formalism, and combines them into a pair of 1-forms:

$$
A_{\mu}^{a}=\frac{1}{2} \epsilon_{b c}^{a} \omega_{\mu}^{b c}+\frac{1}{l} e_{\mu}^{a}, \quad \tilde{A}_{\mu}^{a}=\frac{1}{2} \epsilon_{b c}^{a} \omega_{\mu}^{b c}-\frac{1}{l} e_{\mu}^{a}
$$

where $l$ is a constant with dimensions of length. Taking $T^{a}, a=1,2,3$ to be the generators of $\mathrm{SL}(2, \mathrm{R})$, normalised as $\operatorname{tr}\left(T^{a} T^{b}\right)=\frac{1}{2} \eta^{a b}$, and defining $A_{\mu}=A_{\mu}^{a} T^{a}$, the Lagrangian:

$$
\mathcal{L}=\frac{l}{16 \pi G_{N}} \operatorname{tr}\left(A \wedge d A+\frac{2}{3} A \wedge A \wedge A-\tilde{A} \wedge d \tilde{A}-\frac{2}{3} \tilde{A} \wedge \tilde{A} \wedge \tilde{A}\right)
$$

can be shown to be equivalent to ordinary Einstein gravity in $(2+1)$ d with a negative cosmological constant $\Lambda=-\frac{3}{l^{2}}$.

Except for the choice of a specific non-compact gauge group, $\mathrm{SL}(2, \mathrm{R})$, this is identical to the Lagrangian Eq. (4.1) that we discussed in Section 4.1. It is also natural to write this action in the form of Eq. (4.4) because the fields $B_{\mu}, C_{\mu}$ defined there are, in the present case:

$$
B_{\mu}^{a}=\frac{1}{l} e_{\mu}^{a}, \quad C_{\mu}^{a}=\frac{1}{2} \epsilon_{b c}^{a} \omega_{\mu}^{b c}
$$

This gives a nice physical interpretation in the context of gravity, to the fields $B_{\mu}, C_{\mu}$ of Section 4.1.

We also see that in terms of these fields, the terms $B \wedge d C$ and $B \wedge C \wedge C$ are of order $\frac{1}{l}$ while the term $B \wedge B \wedge B$ is of order $\frac{1}{l^{3}}$. Thus the limit $l \rightarrow \infty$ is easily taken and leads to the pure $B \wedge F^{(C)}$ Lagrangian Eq. 4.27) of Sec. 4.2, with now $B_{\mu}^{a}=e_{\mu}^{a}$ since the $l$-dependence has cancelled against the coefficient of the Lagrangian. This Lagrangian therefore corresponds to $(2+1) \mathrm{d}$ gravity in the absence of a cosmological constant.

It is worth remarking that there is a beautiful generalisation of the above structure to the case of higher-spin fields 26, 27). Here the 1-forms in Eq. (5.11) are generalised to include the corresponding quantities describing one or more higher-spin fields. With this generalisation all the above formulae carry over identically.

It remains to ask whether there is a novel Higgs mechanism in this system. This would require a mass term: $\sim-\operatorname{tr}\left(B_{\mu} B^{\mu}\right)=-\frac{1}{2} \eta^{\mu \nu} \eta_{a b} e_{\mu}{ }^{a} e_{\nu}{ }^{b}$ which is of course not generally covariant. In fact, in the Chern-Simons formulation general covariance is replaced by $\mathrm{SL}(2, \mathrm{R}) \times \mathrm{SL}(2, \mathrm{R})$ gauge invariance, and a mass term will not be gauge-invariant, so this is not necessarily a surprise. However, an additional problem is that the metric $\eta_{a b}$ of $\mathrm{SL}(2, \mathrm{R})$ has Lorentzian signature so the above "mass term" for $e_{\mu}{ }^{a}$ has wrong signs for some components (in our metric, the right sign would be negative for spacelike values of 
$\mu$, but here we get positive signs when $\mu$ is spacelike and $a$ is timelike). Finally, if such a term were nevertheless generated and formally used to integrate out $e_{\mu}^{a}$, the result would be $\mathrm{SL}(2, \mathrm{R})$ Yang-Mills theory which by itself continues to be plagued with sign issues.

It is still (barely) conceivable that a mass term for $e_{\mu}^{a}$ is generated along just one (spacelike) direction of $\mathrm{SL}(2, \mathrm{R})$. This would Higgs a $\mathrm{U}(1) \times \mathrm{U}(1)$ part of the ChernSimons action and lead to a Maxwell kinetic term. We leave this possibility for a future investigation.

\section{Acknowledgements}

I would like to thank Guillaume Bossard, Kedar Damle, Bobby Ezhuthachan, Rajesh Gopakumar, Kimyeong Lee, Shiraz Minwalla, Nitin Nitsure, Costis Papageorgakis, David Tong and particularly Nemani Suryanarayana for helpful discussions. Generous support for the basic sciences by the people of India is gratefully acknowledged.

\section{Notation and conventions}

We work with the $(2+1) d$ metric:

$$
\eta_{\mu \nu}=\operatorname{diag}(-,+,+)
$$

The differential form notation used throughout is easily translated into conventional index notation using the following identites:

$$
\begin{aligned}
A \wedge^{*} A & =-A_{\mu} A^{\mu} \\
A \wedge d A & =\epsilon^{\mu \nu \lambda} A_{\mu} \partial_{\nu} A_{\lambda} \\
d A \wedge^{*} d A & =-\frac{1}{2} F_{\mu \nu} F^{\mu \nu}
\end{aligned}
$$

Finally, in the non-Abelian context with compact groups, we have:

$$
A=A^{a} T^{a}, F=F^{a} T^{a}
$$

where $T^{a}$ are anti-Hermitian, and:

$$
\operatorname{tr} T^{a} T^{b}=-\frac{1}{2} \delta^{a b}
$$

\section{References}

[1] R. Jackiw and S. Templeton, How superrenormalizable interactions cure their infrared divergences, Phys.Rev. D23 (1981) 2291.

[2] J. F. Schonfeld, A mass term for three-dimensional gauge fields, Nucl.Phys. B185 (1981) 157. 
[3] S. Deser, R. Jackiw, and S. Templeton, Topologically massive gauge theories, Ann. Phys. 140 (1982) 372-411.

[4] S. Mukhi and C. Papageorgakis, M2 to D2, JHEP 05 (2008) 085, arXiv:0803.3218.

[5] G. W. Semenoff, Canonical quantum field theory with exotic statistics, Phys.Rev.Lett. 61 (1988) 517.

[6] S. Zhang, T. Hansson, and S. Kivelson, An effective field theory model for the fractional quantum Hall effect, Phys.Rev.Lett. 62 (1988) 82-85.

[7] N. Read, Order parameter and Ginzburg-Landau theory for the fractional quantum Hall effect, Phys.Rev.Lett. 62 (1989) 86-89.

[8] X. G. Wen and A. Zee, Topological structures, universality classes, and statistics screening in the anyon superfluid, Phys. Rev. B 44 (1991) 274-284.

[9] M. A. Bandres, A. E. Lipstein, and J. H. Schwarz, $N=8$ superconformal Chern-Simons theories, JHEP 05 (2008) 025, arXiv:0803.3242.

[10] M. Van Raamsdonk, Comments on the Bagger-Lambert theory and multiple M2- branes, JHEP 05 (2008) 105, arXiv:0803.3803.

[11] S. Deser and R. Jackiw, 'Selfduality' of topologically massive gauge theories, Phys. Lett. B139 (1984) 371.

[12] P. K. Townsend, K. Pilch, and P. van Nieuwenhuizen, Selfduality in odd dimensions, Phys. Lett. 136B (1984) 38.

[13] S. Deser and Z. Yang, A remark on the Higgs effect in presence of Chern-Simons terms, Mod. Phys. Lett. A4 (1989) 2123.

[14] J. Bagger and N. Lambert, Gauge symmetry and supersymmetry of multiple M2-branes, Phys. Rev. D77 (2008) 065008, arXiv:0711.0955.

[15] O. Aharony, O. Bergman, D. L. Jafferis, and J. Maldacena, $N=6$ superconformal Chern-Simons-matter theories, M2-branes and their gravity duals, JHEP 10 (2008) 091, arXiv:0806.1218].

[16] H. Stephani et. al., Exact solutions of Einstein's field equations. Cambridge University Press, UK, 2003.

[17] W. Greub, Linear Algebra. Springer-Verlag, 1963.

[18] W. C. Waterhouse, Pairs of quadratic forms, Inv. Math. 37 (1976) 157.

[19] J. Distler, S. Mukhi, C. Papageorgakis, and M. Van Raamsdonk, M2-branes on M-folds, JHEP 05 (2008) 038, arXiv:0804.1256.

[20] A. Armoni and A. Naqvi, A non-supersymmetric large-N 3D CFT and its gravity dual, JHEP 0809 (2008) 119, arXiv:0806.4068]. 
[21] B. Ezhuthachan, S. Mukhi, and C. Papageorgakis, D2 to D2, JHEP 07 (2008) 041, arXiv:0806.1639.

[22] J. K. Jain, Composite-fermion approach for the fractional quantum hall effect, Phys. Rev. Lett. 63 (1989) 199-202.

[23] M. Greiter and F. Wilczek, Heuristic principle for quantized Hall states, Mod.Phys.Lett. B4 (1990) 1063-1070.

[24] A. Achucarro and P. Townsend, A Chern-Simons action for three-dimensional anti-de Sitter supergravity theories, Phys.Lett. B180 (1986) 89.

[25] E. Witten, (2+1)-dimensional gravity as an exactly soluble system, Nucl.Phys. B311 (1988) 46.

[26] M. Henneaux and S.-J. Rey, Nonlinear $W_{\text {infinity }}$ as asymptotic symmetry of three-dimensional higher spin anti-de Sitter gravity, JHEP 1012 (2010) 007, arXiv:1008.4579.

[27] A. Campoleoni, S. Fredenhagen, S. Pfenninger, and S. Theisen, Asymptotic symmetries of three-dimensional gravity coupled to higher-spin fields, JHEP 1011 (2010) 007, arXiv: 1008.4744 . 